\title{
Differences in Clinical Presentation and Findings between Idiopathic Di- lated and Ischaemic Cardiomyopathy in an Unselected Population of Heart Failure Patients
}

\author{
Lilian Mantziari*, ${ }^{1}$, Antonis Ziakas ${ }^{1}$, Ioannis Ventoulis ${ }^{1}$, Vasileios Kamperidis ${ }^{1}$, Leonidas Lilis ${ }^{1}$, \\ Niki Katsiki $^{3}$, Savvato Karavasiliadou ${ }^{1}$, Konstantinos Kiraklidis ${ }^{1}$, Christodoulos Pliakos ${ }^{2}$, Konstan- \\ tinos Gemitzis ${ }^{1}$, Haralambos Karvounis ${ }^{1}$ and Ioannis H Styliadis ${ }^{1}$ \\ ${ }^{1}$ First Cardiology Department, AHEPA University Hospital, Thessaloniki, Greece \\ ${ }^{2}$ First Department of Internal Medicine, AHEPA University Hospital, Thessaloniki, Greece \\ ${ }^{3}$ Second Propedeutic Department of Internal Medicine, Medical School, Aristotle University of Thessaloniki, Hippocra- \\ tion Hospital, Thessaloniki, Greece
}

\begin{abstract}
We explored the differences in epidemiologic, clinical, laboratory and echocardiographic characteristics between idiopathic dilated (IDCM) and ischaemic cardiomyopathy (ICM).

Consecutive patients with stable chronic heart failure evaluated at a tertiary cardiac centre were enrolled. Clinical examination, blood tests and echocardiographic study were performed.

A total of 76 patients (43 IDCM, 33 ICM) were studied. IDCM patients were younger $(p<0.001)$ and female gender was more prevalent $(\mathrm{p}=0.022)$. NYHA class and left ventricular ejection fraction were similar. IDCM patients had lower rates of dyslipidaemia $(\mathrm{p}<0.001)$ but smoked more than ICM patients $(\mathrm{p}=0.023)$ and had higher rates of family history of sudden cardiac death $(p=0.048)$. Blood pressure was similar but resting heart rate was higher in IDCM patients $(p=0.022)$. IDCM patients presented less frequently with peripheral oedema or ascites ( $\mathrm{p}=0.046$ and 0.020 , respectively) and showed better right ventricular function on echocardiogram. QRS duration was similar between groups but only in IDCM patients there was a positive correlation between QRS duration and age $(\mathrm{r}=0.619, \mathrm{p}<0.001)$. Cardiac output was similar but functional capacity assessed by the Duke Activity Status Index was better in IDCM ( $p=0.036)$. Despite these differences, IDCM and ICM patients received similar treatments.

Patients with IDCM were younger, presented lower rates of right ventricular dysfunction and clinical right ventricular failure and had better functional capacity. Additional differences in clinical and laboratory findings exist pointing to a different patient population with diverse prognosis and potential need for individualized management.
\end{abstract}

Keywords: Dyslipidaemia, echocardiogram, idiopathic dilated cardiomyopathy, ischaemic cardiomyopathy, right ventricular dysfunction, smoking.

\section{INTRODUCTION}

Idiopathic dilated (IDCM) or ischaemic cardiomyopathy (ICM) are among the main causes of chronic heart failure (CHF) in Western countries [1]. Both diagnoses require evidence of dilatation and impaired contractility of the left or both ventricles and the diagnosis of IDCM is based on exclusion of more than $50 \%$ obstruction of $\geq 1$ coronary arteries on angiography, active myocarditis or other heart muscle disease [2]. Apart from correcting ischaemia with revascularization which may improve ICM, no other aetiology-guided treatment is usually applied according to current guidelines

*Address correspondence to this author at the First Cardiology Department, AHEPA University Hospital, Thessaloniki, St. Kiriakidi 1, 54636, Greece; Tel: +30 2310 994830; Fax: +30 2310 994673;

E-mails: 1mantziari@yahoo.com, 1mantziari@gmail.com and clinical practice $[3,4]$. However, both these conditions have been associated with differential characteristics and prognosis $[5,6]$. Patients with IDCM showed better right ventricular function by tissue Doppler imaging [7] and less severe functional mitral regurgitation [8] compared with patients with ischaemic disease. Further differences in the prevalence and location of endomyocardial fibrosis [9] and in ventricular tachycardia substrate [10] exist between IDCM and ICM.

We explored the differences between IDCM and ICM in epidemiologic characteristics, clinical presentation, echocardiographic and routine laboratory findings in an unselected population of patients with heart failure evaluated at a tertiary cardiac centre. 


\section{METHODS}

\section{Patient Population}

Consecutive patients with stable CHF followed up at a tertiary cardiac centre were screened for eligibility for a prospective observational study designed to evaluate newer indices for the assessment of IDCM. Inclusion criteria were age $>18$ years, ejection fraction $<45 \%$ and history of known CHF because of ICM or IDCM. Non-ischaemic dilated cardiomyopathy was diagnosed after exclusion of severe coronary artery disease (causing $>50 \%$ stenosis in $\geq 1$ coronary arteries) by coronary angiography or exclusion of ischaemia and scar by nuclear perfusion scan in 2 cases of young patients who refused to undergo coronary angiography. Furthermore, diagnosis of idiopathic dilated cardiomyopathy was done after exclusion of active myocarditis and other causes of non-ischaemic cardiomyopathy i.e. hypertensive heart disease, valvular heart disease, infiltrative diseases, cardiomyopathies associated with connective tissue diseases, peri-partum cardiomyopathy, tachyarrhythmias, thyroid dysfunction and congenital heart disease. Exclusion criteria were recent ( $\leq 30$ days) acute coronary syndrome, pulmonary oedema or decompensation of heart failure, permanent pacemakers or cardiac resynchronization devices, malignancy and severe renal failure requiring haemofiltration. The study protocol was approved by the ethics committee of our institution and all subjects gave written informed consent.

\section{Clinical Evaluation and Laboratory Tests}

Clinical evaluation included a detailed personal and family medical history, standard clinical examination, electrocardiogram (ECG) and review of all drugs. Furthermore, all patients completed a translated questionnaire for the Duke activity status index (DASI) calculation [11]. DASI is a 12point scale for the assessment of functional capacity based on the ability to perform everyday activities [11] and has been previously validated in patients with heart failure [12, 13]. Clinical evaluation was done by 2 study investigators (LM and IV) 1-2 days apart from the echocardiographic study. Full blood count and standard biochemical tests were performed. Estimated glomerular filtration rate (eGFR) was calculated using the Cockroft-Gault formula (http://clinicalculator.com/english/nephrology/cockroft/cca.htm).

\section{Echocardiographic Study}

A full echocardiographic study was performed by a single European Society of Cardiology (ESC) accredited echocardiographer to all study subjects using the same equipment (Vivid 7, GE Vingmed Ultrasound, Horten, Norway). The echocardiographer was a study investigator (VK) and performed the echocardiograms unaware of the clinical details and all measurements were done off-line. Standard echocardiographic views were used. Left ventricular dimensions were measured in $2 \mathrm{D}$ images at the long parasternal axis. Left ventricular volumes and ejection fraction (EF) were calculated using the biplane Simpson's method from 4 and 2 chamber views at the apical axis. Atrial volumes and right ventricular dimensions were calculated from the 4-chamber view. RV dimensions and function were evaluated according to established methods [14]. Tricuspid annular plane systolic excursion (TAPSE) was measured by 2-dimensional difference of end-diastolic and end-systolic lines (in $\mathrm{cm}$ ) traced between the centre of the ultrasound fan origin and the junction of right ventricular lateral tricuspid annulus, in apical 4chamber view. Right ventricular systolic pressure was estimated by calculating the maximal velocity of the tricuspid regurgitant jet and then, further using the Bernoulli equation, adding to this value an estimated right atrial pressure based on both the size of the inferior vena cava and the change in diameter of this vessel during respiration. Pulsed Doppler echocardiography for the assessment of the standard diastolic filling velocities of both ventricles was performed using the apical 4-chamber view. Thus, the peak early diastolic filling velocity (E-wave), peak late diastolic filling velocity (Awave) and their ratio (E/A) were recorded. Pulsed-wave Tissue Doppler imaging (TDI) was used to assess mitral and tricuspid annular velocities. Filters were set to exclude highfrequency signals, and the Nyquist limit was adjusted to a velocity range of $15-20 \mathrm{~cm} / \mathrm{s}$. Gains were minimized in order to allow for a clear tissue signal with minimum background noise. All TDI recordings were obtained during normal respiration, in the apical 4 chamber view. A 5-mm sample volume was placed at the septal and lateral corner of the mitral annulus and at the lateral corner of the tricuspid annulus. The peak myocardial velocities during systole (S'), early diastole (E'), late diastole (A') and their ratio (E'/A') were recorded at a sweep speed of $100 \mathrm{~mm} / \mathrm{s}$. Furthermore, E to E' ratio was calculated for both ventricles.

Cardiac output was calculated using the formula: $Q=(\pi$ $\mathrm{x}$ HR $\left.x \mathrm{LVOT}^{2} \times \mathrm{VTI}\right) / 4$, where LVOT is the diameter $(\mathrm{cm})$ of the left ventricular outflow tract measured in the parasternal long-axis view and VTI is the velocity time integral $(\mathrm{cm})$ of the aortic flow measured in the LVOT using pulsed-wave Doppler. Cardiac index was then derived as Cardiac output/Body surface area (BSA). BSA was calculated using the DuBois and DuBois formula (http://www.globalrph.com/ bsa2.cgi).

\section{Statistical Analysis}

Statistical analysis was performed using SPSS v16.0 for windows (Chicago, IL, USA). All variables were checked for normal distribution with Kolmogorov-Smirnov test. Continuous variables were expressed as mean and standard deviation (SD) and categorical variables were expressed as percentages. Differences between groups were determined using the student's t-test or Mann-Whitney $U$ test for normally or non-normally distributed continuous variables, respectively and with chi-square test for categorical variables. Correlations between variables were tested with Pearson's $r$ test. A two-sided $\mathrm{p}<0.05$ was considered significant.

\section{RESULTS}

\section{Epidemiological and Clinical Findings}

A total of 76 patients were included in the final analysis, 43 with IDCM and 33 with ICM. The differences in epidemiologic characteristics and medical history between IDCM and ICM patients are listed in Table 1. Mean age was lower in IDCM patients $(56.7 \pm 14.9$ years vs $71.1 \pm 8.0, \mathrm{p}<0.001)$ and female gender more frequent ( $31 \mathrm{vs} 9 \%, \mathrm{p}=0.022$ ). 
New York Heart Associtation (NYHA) class at presentation and left ventricular EF did not differ significantly between groups. There was a trend for lower prevalence of diabetes mellitus in IDCM patients (22.5 vs $40.6 \%, p=0.097)$. IDCM patients showed lower prevalence of dyslipidaemia and family history of coronary artery disease ( $17.5 \mathrm{vs} 60 \%, \mathrm{p}<0.001$ and 12.5 vs $28.1 \%, \mathrm{p}=0.046$, respectively) but higher rates of smoking ( 25 vs $6.2 \%, p=0.023$ ) and family history of sudden cardiac death ( 18.9 vs $3.2 \%, \mathrm{p}=0.048)$.

Regarding clinical findings (Table 2) and clinical presentation (Fig. 1), there were some noteworthy differences observed. Patients with IDCM had higher resting heart rate (77 \pm 15 vs $70 \pm 9 \mathrm{bpm}, \mathrm{p}=0.022)$ and presented less frequently with peripheral oedema (10.3 vs $31.2 \%, \mathrm{p}=0.046)$ and ascites ( 0 vs $16.1 \%, p=0.020)$. In contrast, blood pressure was similar between groups and the prevalence of atrial fibrillation and left bundle branch block did not differ significantly. QRS duration was also similar between groups (129 \pm 41 vs
$122 \pm 45 \mathrm{~ms}, \mathrm{p}=0.506)$. Of note in IDCM patients QRS duration showed a positive correlation with age $(\mathrm{r}=0.619$, $\mathrm{p}$ $<0.001)$ a finding that was not reproduced in ICM patients (Fig. 2). Serum creatinine levels were comparable between IDCM and ICM patients $(1.15 \pm 0.39$ vs $1.28 \pm 0.61 \mathrm{mg} / \mathrm{dL}$, $\mathrm{p}=0.252$ ); estimated GFR showed a trend for being higher in IDCM patients $(86 \pm 47 \mathrm{vs} 69 \pm 35 \mathrm{ml} / \mathrm{min}, \mathrm{p}=0.084)$. Drug therapies are shown in Fig. (3). Beta-blocker, angiotensin converting enzyme or angiotensin II receptor blocker (ACEi/ARBs), diuretic and aldosterone antagonist prescription was similar between IDCM and ICM. IDCM patients less frequently received digoxin, statins, aspirin and clopidogrel.

\section{Echocardiographic Findings}

Standard echocardiographic findings in IDCM and ICM are shown in Table 3. Apart from thinner interventricular

Table 1. Differences in Epidemiologic Characteristics and Medical History between Patients with Idiopathic Dilated (IDCM) and Ischaemic Cardiomyopathy (ICM)

\begin{tabular}{|c|c|c|c|}
\hline & $\begin{array}{l}\text { IDCM } \\
n=43\end{array}$ & $\begin{array}{c}\text { ICM } \\
\mathbf{n}=\mathbf{3 3}\end{array}$ & $\mathbf{P}$ \\
\hline Age & $56.7 \pm 14.9$ & $71.1 \pm 8.0$ & $<0.001$ \\
\hline Female gender $\%$ & 31 & 9 & 0.022 \\
\hline NYHA class & $2.6 \pm 0.7$ & $2.8 \pm 0.6$ & 0.170 \\
\hline $\mathrm{EF} \%$ & $28.7 \pm 7.8$ & $27.8 \pm 5.6$ & 0.572 \\
\hline Hypertension \% & 50 & 40.6 & 0.428 \\
\hline Diabetes $\%$ & 22.5 & 40.6 & 0.097 \\
\hline Dyslipidaemia \% & 17.5 & 60 & $<0.001$ \\
\hline Family history of CAD \% & 12.5 & 28.1 & 0.046 \\
\hline Alcohol \% & 12.5 & 6.5 & 0.396 \\
\hline Smoking \% & 25 & 6.2 & 0.023 \\
\hline Weight $(\mathrm{Kg})$ & $79.3 \pm 21.4$ & $81.0 \pm 13.2$ & 0.666 \\
\hline Height (m) & $1.70 \pm 0.09$ & $1.70 \pm 0.09$ & 0.772 \\
\hline BMI $\left(\mathrm{Kg} / \mathrm{m}^{2}\right)$ & $27.5 \pm 6.5$ & $28.3 \pm 3.5$ & 0.534 \\
\hline Family history of SCD \% & 18.9 & 3.2 & 0.048 \\
\hline Family history of DCM \% & 5.6 & 3.2 & 0.361 \\
\hline
\end{tabular}

NYHA, New York Heart Association; EF, ejection fraction; CAD, coronary artery disease; BMI, body mass index; SCD, sudden cardiac death; DCM, dilated cardiomyopathy

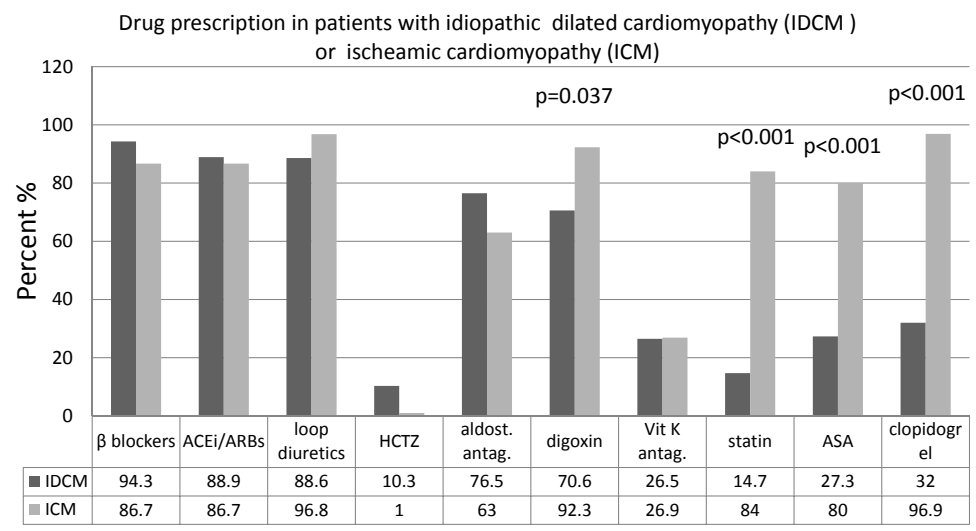

Fig. (1). Clinical presentation of patients with idiopathic dilated cardiomyopathy (IDCM) or ischaemic cardiomyopathy (ICM). 
Table 2. Characteristics of Patients with Idiopathic Dilated (IDCM) or Ischaemic Cardiomyopathy (ICM)

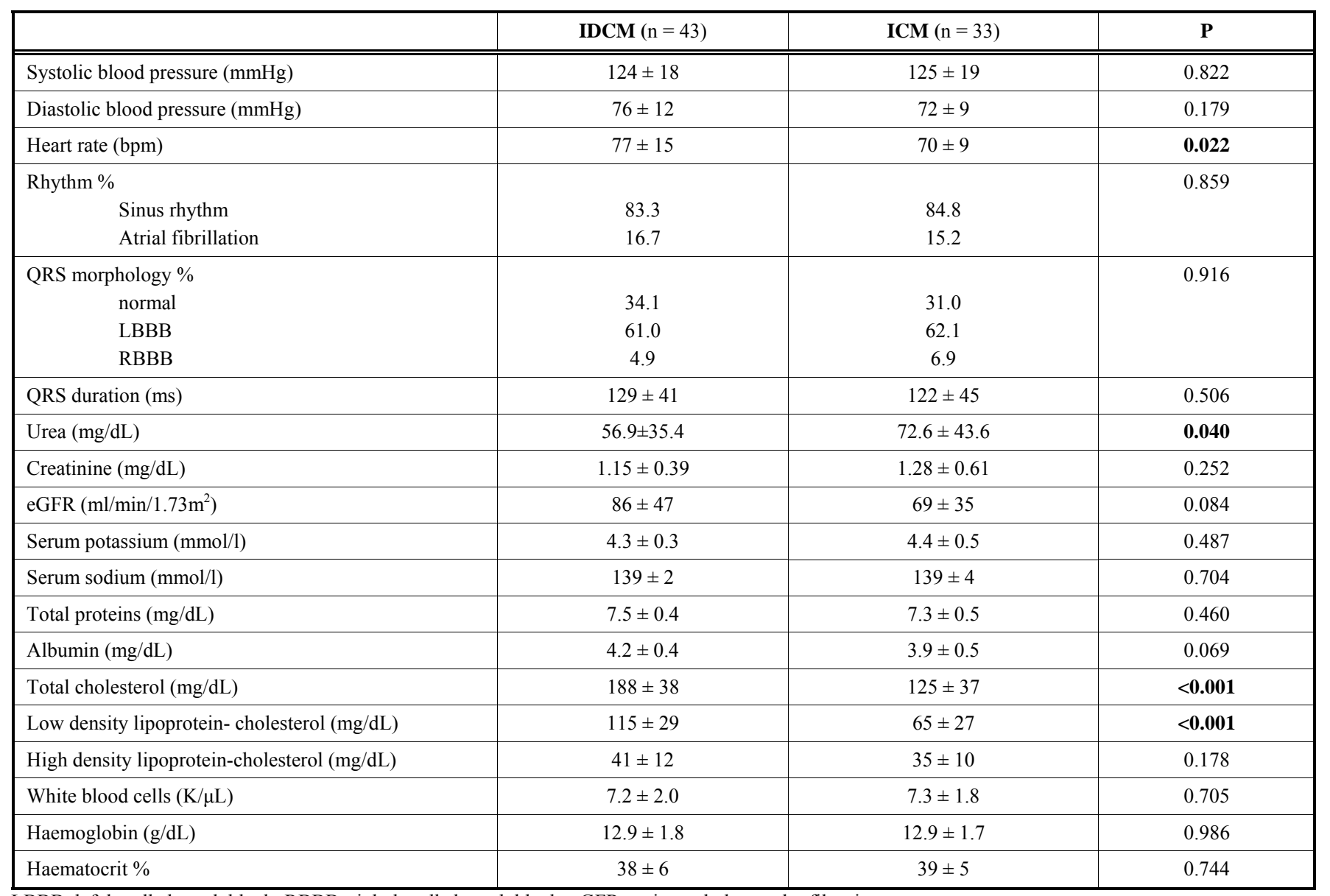

LBBB, left bundle branch block; RBBB, right bundle branch block; eGFR, estimated glomerular filtration rate
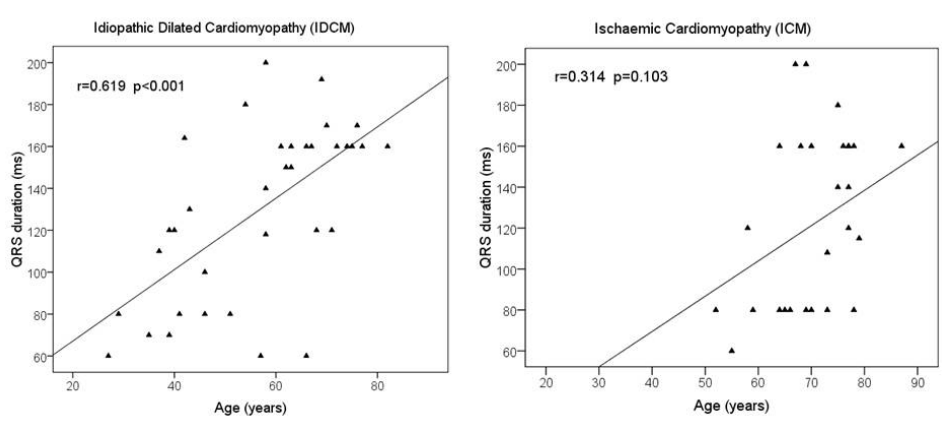

Fig. (2). Correlation between age and QRS duration in patients with idiopathic dilated cardiomyopathy (left panel) and ischaemic cardiomyopathy (right panel).

septum $(0.9 \pm 0.3$ vs $1.1 \pm 0.3 \mathrm{~cm}, \mathrm{p}=0.015)$ no other significant difference was observed in dimensions, volumes and EF of the left ventricle. Left atrial dimensions and volume were also similar. In contrast, patients with IDCM had smaller right ventricular basal and mid diameters $(3.7 \pm 0.8$ vs $4.3 \pm 1.0 \mathrm{~cm}, \mathrm{p}=0.007$ and $2.7 \pm 0.7$ vs $3.1 \pm 0.7 \mathrm{~cm}, \mathrm{p}=$ $0.012)$ and higher TAPSE $(1.9 \pm 0.5$ vs $1.6 \pm 0.4 \mathrm{~cm}, \mathrm{p}=$ $0.001)$. Right atrial volume was smaller in patients with IDCM $(49 \pm 22$ vs $65 \pm 35 \mathrm{ml}, \mathrm{p}=0.022)$. TDI velocities (Table 4) recorded in the lateral portion of the tricuspid annulus were also higher in IDCM patients (RV S', $10.4 \pm 2.9$ vs $8.6 \pm 2.6 \mathrm{~cm} / \mathrm{s}, \mathrm{p}=0.010 ; \mathrm{RV}$ E', $9.5 \pm 3.8$ vs $7.7 \pm 2.6$ $\mathrm{cm} / \mathrm{s}, \mathrm{p}=0.033 ; \mathrm{RV} \mathrm{A}, 11.7 \pm 5.0$ vs $9.0 \pm 3.9 \mathrm{~cm} / \mathrm{s}, \mathrm{p}=$ 0.033). TDI velocities recorded in the LV lateral and septal wall were similar between groups, except for the septal E' which was better in IDCM $(5.2 \pm 1.9$ vs $4.2 \pm 2.0 \mathrm{~cm} / \mathrm{s}, \mathrm{p}=$ 0.038).

\section{Cardiac Output and Functional Capacity}

Cardiac output and cardiac index (Table 5) did not differ in DCM and ICM patients $(4.1 \pm 1.7$ vs $3.9 \pm 1.3, \mathrm{p}=0.683$ and $2.3 \pm 1.3$ vs $2.1 \pm 0.7, p=0.436)$. However the DASI 
Table 3. Differences in Standard Echocardiographic Findings between Patients with Idiopathic Dilated Cardiomyopathy (IDCM) and Ischaemic Cardiomyopathy (ICM)

\begin{tabular}{|c|c|c|c|}
\hline & $\begin{array}{l}\text { IDCM } \\
n=43\end{array}$ & $\begin{array}{c}\text { ICM } \\
\mathbf{n}=\mathbf{3 3}\end{array}$ & $\mathbf{P}$ \\
\hline Interventricular septum $(\mathrm{cm})$ & $0.9 \pm 0.3$ & $1.1 \pm 0.3$ & 0.015 \\
\hline LV posterior wall $(\mathrm{cm})$ & $1.0 \pm 0.2$ & $1.1 \pm 1.9$ & 0.379 \\
\hline $\mathrm{LV}$ end diastolic diameter $(\mathrm{cm})$ & $6.5 \pm 0.8$ & $6.6 \pm 0.8$ & 0.822 \\
\hline LV end systolic diameter $(\mathrm{cm})$ & $5.7 \pm 1.2$ & $5.8 \pm 0.9$ & 0.554 \\
\hline LV end diastolic volume (ml) & $140 \pm 58$ & $142 \pm 51$ & 0.876 \\
\hline LV ejection fraction $(\%)$ & $28.7 \pm 7.8$ & $27.8 \pm 5.7$ & 0.572 \\
\hline LVOT diameter $(\mathrm{cm})$ & $2.1 \pm 0.3$ & $2.2 \pm 0.2$ & 0.352 \\
\hline Left atrial diameter $(\mathrm{cm})$ & $4.7 \pm 0.7$ & $4.9 \pm 0.5$ & 0.399 \\
\hline Left atrial volume $(\mathrm{ml})$ & $95 \pm 48$ & $95 \pm 38$ & 0.963 \\
\hline Left atrial volume index $\left(\mathrm{ml} / \mathrm{m}^{2}\right)$ & $51.6 \pm 26.2$ & $50.3 \pm 21.0$ & 0.835 \\
\hline Right atrial volume (ml) & $49 \pm 22$ & $65 \pm 35$ & 0.022 \\
\hline Right atrial volume index $\left(\mathrm{ml} / \mathrm{m}^{2}\right)$ & $26.5 \pm 13.7$ & $34.1 \pm 18.3$ & 0.056 \\
\hline RV basal diameter $(\mathrm{cm})$ & $3.7 \pm 0.8$ & $4.3 \pm 1.0$ & 0.007 \\
\hline RV mid diameter $(\mathrm{cm})$ & $2.7 \pm 0.7$ & $3.1 \pm 0.7$ & 0.012 \\
\hline TAPSE (cm) & $1.9 \pm 0.5$ & $1.6 \pm 0.4$ & 0.001 \\
\hline RVOT (cm) & $2.8 \pm 0.7$ & $2.8 \pm 0.6$ & 0.757 \\
\hline Pulmonary valve $V \max (\mathrm{m} / \mathrm{s})$ & $0.9 \pm 0.2$ & $0.9 \pm 0.2$ & 0.955 \\
\hline Tricuspid regurgitation $V \max (\mathrm{m} / \mathrm{s})$ & $2.9 \pm 0.7$ & $2.9 \pm 0.7$ & 0.984 \\
\hline Tricuspid regurgitation $\mathrm{PG}(\mathrm{mmHg})$ & $34.7 \pm 18.6$ & $35.2 \pm 15.7$ & 0.918 \\
\hline Aortic valve $V \max (\mathrm{m} / \mathrm{s})$ & $1.3 \pm 0.4$ & $1.2 \pm 0.4$ & 0.264 \\
\hline Mitral regurgitation Vmax $(\mathrm{m} / \mathrm{s})$ & $4.1 \pm 1.2$ & $4.1 \pm 0.8$ & 0.911 \\
\hline Mitral E (m/s) & $0.90 \pm 0.28$ & $0.81 \pm 0.38$ & 0.284 \\
\hline Mitral A (m/s) & $0.66 \pm 0.29$ & $0.60 \pm 0.31$ & 0.537 \\
\hline Mitral E/A & $1.6 \pm 1.0$ & $1.8 \pm 1.1$ & 0.696 \\
\hline Mitral deceleration time (ms) & $173 \pm 68$ & $179 \pm 72$ & 0.733 \\
\hline Tricuspid E (m/s) & $0.47 \pm 0.15$ & $0.44 \pm 0.15$ & 0.505 \\
\hline Tricuspid A (m/s) & $0.40 \pm 0.16$ & $0.44 \pm 0.17$ & 0.446 \\
\hline Tricuspid deceleration time (ms) & $214 \pm 77$ & $219 \pm 87$ & 0.835 \\
\hline Tricuspid E/A & $1.3 \pm 0.5$ & $1.1 \pm 0.4$ & 0.157 \\
\hline LV restrictive filling pattern $(E / A \geq 2) \%$ & 17.0 & 18.9 & 0.750 \\
\hline Inferior vena cava diameter (cm) & $1.69 \pm 0.54$ & $1.85 \pm 0.53$ & 0.250 \\
\hline Right atrial pressure $(\mathrm{mmHg})$ & $9.8 \pm 5.7$ & $11.2 \pm 5.4$ & 0.352 \\
\hline Right ventricular systolic pressure (mmHg) & $44.9 \pm 21.9$ & $46.6 \pm 18.9$ & 0.769 \\
\hline
\end{tabular}

LV, left ventricular; LVOT, left ventricular outflow tract; RV, right ventricular; TAPSE, tricuspid annular plane systolic excursion; RVOT, right ventricular outflow tract; Vmax, maximum velocity; PG, peak gradient; E, peak early diastolic filling velocity; A, peak late diastolic filling velocity; E/A, peak early and late diastolic filling velocity ratio

scores were higher for IDCM patients $(24.6 \pm 15.8$ vs $16.9 \pm$ 14.8, $\mathrm{p}=0.036)$.

\section{DISCUSSION}

Our data suggests that differences in epidemiological characteristics in clinical presentation, in laboratory findings and in echocardiographic parameters exist between IDCM and ICM.
Patients with IDCM were younger and the prevalence of female gender was higher. As expected the rates of coronary artery disease risk factors (dyslipidaemia, diabetes, history of CAD) were lower in IDCM patients but IDCM patients were more frequently smokers. This may have resulted from the greater percentage of ICM patients who had quit smoking for secondary prevention. Family history of sudden cardiac death was $19 \%$ in the IDCM population while the percentage of documented familial IDCM was $5.6 \%$. This finding may reflect the underdiagnosis of familial IDCM. It is believed 
Table 4. Differences in Pulsed-wave Tissue Doppler (TDI) Measurements between Patients with Idiopathic Dilated Cardiomyopathy (IDCM) or Ischaemic Cardiomyopathy (ICM)

\begin{tabular}{|c|c|c|c|}
\hline & $\begin{array}{l}\text { IDCM } \\
\mathrm{n}=43\end{array}$ & $\begin{array}{c}\text { ICM } \\
\mathrm{n}=33\end{array}$ & $\mathbf{P}$ \\
\hline Lateral S' (cm/s) & $5.3 \pm 3.8$ & $6.9 \pm 3.2$ & 0.258 \\
\hline Lateral E' $(\mathrm{cm} / \mathrm{s})$ & $7.2 \pm 3.8$ & $6.9 \pm 3.2$ & 0.753 \\
\hline Lateral A' (cm/s) & $6.1 \pm 2.5$ & $5.3 \pm 2.2$ & 0.210 \\
\hline Septal E' $(\mathrm{cm} / \mathrm{s})$ & $5.2 \pm 1.9$ & $4.2 \pm 2.0$ & 0.038 \\
\hline Septal A' $(\mathrm{cm} / \mathrm{s})$ & $5.7 \pm 2.5$ & $4.7 \pm 1.8$ & 0.084 \\
\hline Lateral E/E' & $16.7 \pm 11.4$ & $13.6 \pm 8.0$ & 0.213 \\
\hline Septal E/E & $19.4 \pm 8.3$ & $21.9 \pm 12.7$ & 0.345 \\
\hline Right ventricular S' $(\mathrm{cm} / \mathrm{s})$ & $10.4 \pm 2.9$ & $8.6 \pm 2.6$ & 0.010 \\
\hline Right ventricular E' $(\mathrm{cm} / \mathrm{s})$ & $9.5 \pm 3.8$ & $7.7 \pm 2.6$ & 0.033 \\
\hline Right ventricular A' $(\mathrm{cm} / \mathrm{s})$ & $11.7 \pm 5.0$ & $9.0 \pm 3.9$ & 0.033 \\
\hline Right ventricular E/E' & $6.0 \pm 2.9$ & $6.7 \pm 2.9$ & 0.460 \\
\hline Right ventricular E'/A' & $0.9 \pm 0.6$ & $1.0 \pm 0.6$ & 0.839 \\
\hline
\end{tabular}

S', peak myocardial velocities during systole; E', early diastole; A', late diastole; E'/A', early and late diastole ratio

Clinical presentation of patients with idiopathic dilated cardiomyopathy (IDCM) or ischaemic cardiomyopathy (ICM)

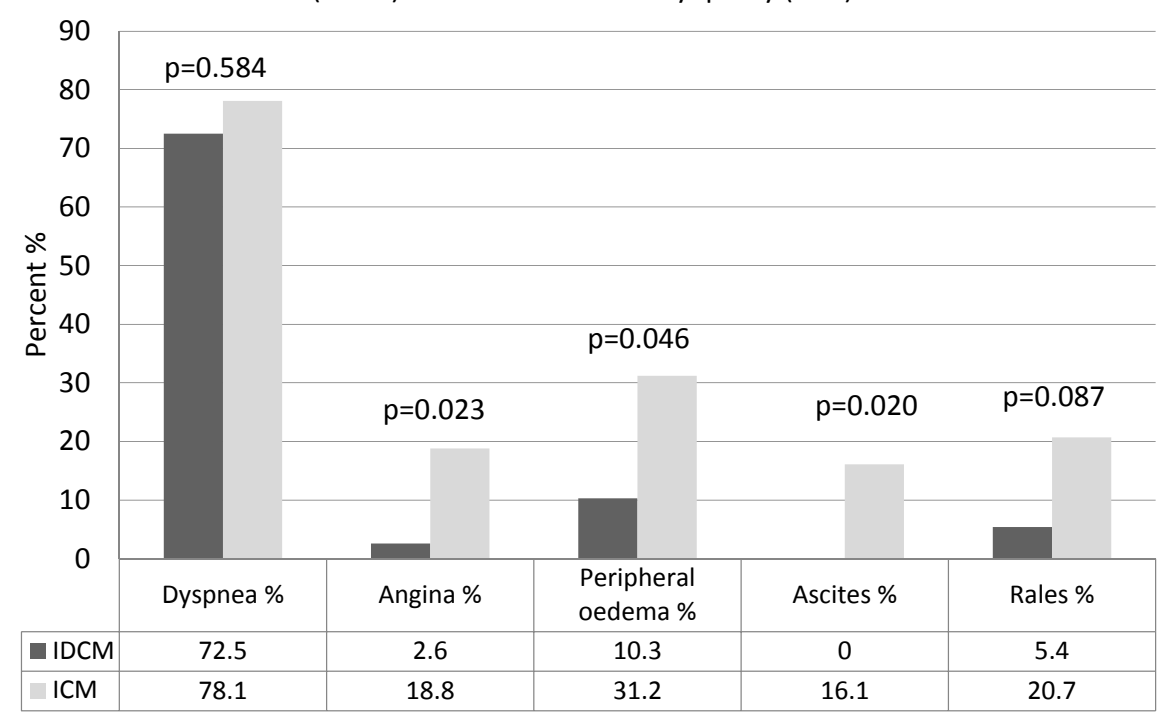

Fig. (3). Rates of prescription of heart failure drug therapies in patients with idiopathic dilated cardiomyopathy (IDCM) or ischaemic cardiomyopathy (ICM). Only significant $\mathrm{p}$ values are shown.

ACEi/ARBs, angiotensin converting enzyme inhibitors or angiotensin II receptor blockers; HTCZ, hydrochlorothiazide; aldost.antag, aldosterone antagonists; Vit K antag., Vitamin K antagonists; ASA, acetylsalicylic acid

that there is a genetic basis in approximately $20-50 \%$ of IDCM cases and screening with echocardiography of firstdegree relatives of probands has revealed that $20-48 \%$ have affected relatives [15-17].
Even though the rate of b-blocker prescription was similar between IDCM and ICM, resting heart rate was higher in IDCM patients. There may be several reasons for this observation. First, Bristow et al. found that although downregula- 
Table 5. Cardiac Output and Duke Activity Status Index Score in Patients with Idiopathic Dilated Cardiomyopathy (IDCM) and Ischaemic Cardiomyopathy (ICM).

\begin{tabular}{|c|c|c|c|}
\hline & $\begin{array}{l}\text { IDCM } \\
n=43\end{array}$ & $\begin{array}{c}\text { ICM } \\
\mathbf{n}=33\end{array}$ & $\mathbf{P}$ \\
\hline LV outflow tract velocity-time interval (LVOT-VTI)(ms) & $15.5 \pm 4.5$ & $15.9 \pm 4.1$ & 0.722 \\
\hline Cardiac output (ml/min) & $4.1 \pm 1.7$ & $3.9 \pm 1.3$ & 0.683 \\
\hline Cardiac index $\left(\mathrm{ml} / \mathrm{min} / \mathrm{m}^{2}\right)$ & $2.3 \pm 1.3$ & $2.1 \pm 0.7$ & 0.436 \\
\hline Duke Activity Status index (DASI) & $24.6 \pm 15.8$ & $16.9 \pm 14.8$ & 0.036 \\
\hline
\end{tabular}

tion of total $\beta$ and $\beta 1$-receptors is greater in IDCM patients they exhibit less blunting of $\beta$-agonist-mediated adenylate cyclase stimulation [18]. Second, ICM patients were older and there has been described a decrease in cardiac betaadrenergic responsiveness with aging in non-failing human hearts [19]. Last, ICM patients were treated more often with digoxin on top of $\beta$-blocker therapy.

Clinical presentation with right heart failure and right ventricular dysfunction were more common among patients with ICM. This finding is in line with a previous study in a similar unselected population of CHF patients which showed that IDCM patients had better right ventricular function assessed by Doppler imaging [7]. However, D' Andrea et al. found that IDCM patients without clinical signs of right heart failure had worse right ventricular function using $2 \mathrm{D}$ strain analysis compared with ICM patients [20]. A possible explanation for this inconsistency is that D' Andrea et al. [20] studied a group of patients referred for cardiac resynchronization therapy with similar age while our study included an unselected population of consecutive CHF patients. Hence, IDCM patients were younger and even though they did not differ in terms of NYHA class and EF from the ICM patients, there seems to be a longer early stage in the progression of the disease when right ventricular function is not yet impaired. Similarly, the observed trend for better eGFR and the better functional capacity assessed by the DASI score in IDCM patients may reflect the younger age and the earlier stage of CHF of IDCM patients regularly followed up in our institution.

QRS prolongation is an established marker of left ventricular dysfunction and adverse prognosis in patients with CHF [21]. We showed a positive correlation between age and QRS duration in IDCM patients implying a mechanism of progression of QRS prolongation with aging. Xiao et al demonstrated in patients with dilated cardiomyopathy of various aetiologies that there was a $5 \mathrm{~ms}$ annual prolongation of QRS duration and larger increases were associated with higher mortality [22]. In contrast, in healthy subjects from the Framingham study, there was a narrowing of the QRS complex associated with aging in men but only a leftward axis shift in women [23]. The lack of association of QRS duration and age in ICM patients we observed is probably due to the underrepresentation of younger patients with ICM but to the different natural course of the ischaemic cardiomyopathy as well. There is one study demonstrating that
QRS duration was longer in patients early after a myocardial infarction compared with patients late ( $>9$ months) after a myocardial infarction [24].

Statins have been reported to beneficially affect inflammatory parameters and left ventricular function in DCM patients $[25,26]$. However, in the present study, only $14.7 \%$ of IDCM patients received statins, most probably due to their low rates of dyslipidaemia (17.5\%). In contrast, $84 \%$ of ICM patient presented to the tertiary center on statin therapy, reflecting a real clinical setting; as expected this percentage is higher than for the IDCM patients.

Anaemia has been associated with increased long-term mortality in CHF patients; in a previous study mild anaemia (defined as haemoglobin $<12 \mathrm{~g} / \mathrm{dl}$ in women and $<13 \mathrm{~g} / \mathrm{dl}$ in men) was significant during the first 2 years, whereas moderate anaemia (defined as haemoglobin $<11 \mathrm{~g} / \mathrm{dl}$ in women and $<12 \mathrm{~g} / \mathrm{dl}$ in men) remained significant for at least 5 years [27]. In the present study, no patient had anaemia.

The present study has limitations. First, the number of patients studied is small. Second, we measured functional capacity using a questionnaire method (Duke Activity Status Index). Cardiopulmonary stress test was not performed in order to minimize cost and the DASI has been validated and found to correlate well with $\mathrm{VO}_{2}$ max [28]. Last, right ventricular function is difficult to quantify by echocardiography as volume calculation and EF measurements are not accurate. We used myocardial wall motion measurements and transvalvular velocities, by M-mode, conventional Doppler and tissue Doppler velocities which are probably the best objective and reproducible methods in clinical practice [29]. Moreover, all measurements were performed by the same experienced echocardiographer.

In conclusion, there are differences in epidemiological characteristics, clinical findings and echocardiographic parameters between patients with IDCM or ICM that presented consecutively for evaluation at a tertiary centre. Patients with IDCM present at younger age and although they do not differ in terms of NYHA class and EF they demonstrate better right ventricular function and better functional capacity. However both IDCM and ICM patients received the same heart failure treatments.

\section{DISCLOSURES}

There is no conflict of interest to declare. 


\section{ACKNOWLEDGEMENT}

None declared.

\section{CONFLICT OF INTEREST}

The authors confirm that this article content has no conflicts of interest.

\section{REFERENCES}

[1] Cowie MR, Wood DA, Coats AJS, et al. Incidence and aetiology of heart failure; a population-based study. Eur Heart J 1999; 20: 4218 .

[2] Libby P, Bonow RO, Mann DL, et al. Braunwald's heart disease: A textbook of cardiovascular medicine. $8^{\text {th }}$ ed. Philadephia: Saunders Elsevier 2008.

[3] Dickstein K, Cohen-Solal A, Filippatos G, et al. ESC Guidelines for the diagnosis and treatment of acute and chronic heart failure 2008: the Task Force for the Diagnosis and Treatment of Acute and Chronic Heart Failure 2008 of the European Society of Cardiology. Developed in collaboration with the Heart Failure Association of the ESC (HFA) and endorsed by the European Society of Intensive Care Medicine (ESICM). Eur Heart J 2008; 29: 2388-442.

[4] Fonarow GC, Yancy CW, Albert NM, et al. Heart failure care in the outpatient cardiology practice setting: findings from IMPROVE HF. Circ Heart Fail 2008; 1: 98-106.

[5] Frazier CG, Alexander KP, Newby LK, et al. Associations of gender and etiology with outcomes in heart failure with systolic dysfunction: a pooled analysis of 5 randomized control trials. J Am Coll Cardiol 2007; 49: 1450-8.

[6] Pecini R, Moller DV, Torp-Pedersen C, et al. Heart failure etiology impacts survival of patients with heart failure. Int J Cardiol 2011; 149: 211-5.

[7] Parcharidou DG, Giannakoulas G, Efthimiadis GK, et al. Right ventricular function in ischemic or idiopathic dilated cardiomyopathy. Circ J 2008; 72: 238-44.

[8] Papadopoulou K, Giannakoulas G, Karvounis H, et al. Differences in echocardiographic characteristics of functional mitral regurgitation in ischaemic versus idiopathic dilated cardiomyopathy: a pilot study. Hellenic J Cardiol 2009; 50: 37-44.

[9] McCrohon JA, Moon JC, Prasad SK, et al. Differentiation of heart failure related to dilated cardiomyopathy and coronary artery disease using gadolinium-enhanced cardiovascular magnetic resonance. Circulation 2003; 108: 54-9.

[10] Hsia HH, Callans DJ, Marchlinski FE. Characterization of endocardial electrophysiological substrate in patients with nonischemic cardiomyopathy and monomorphic ventricular tachycardia. Circulation 2003; 108: 704-10.

[11] Hlatky MA, Boineau RE, Higginbotham MB, et al. A brief selfadministered questionnaire to determine functional capacity (the Duke Activity Status Index). Am J Cardiol 1989; 64: 651-4.

[12] Parissis JT, Nikolaou M, Birmpa D, et al. Clinical and prognostic value of Duke's Activity Status Index along with plasma B-type natriuretic peptide levels in chronic heart failure secondary to ischemic or idiopathic dilated cardiomyopathy. Am J Cardiol 2009; 103: 73-5.

[13] Koch CG, Khandwala F, Cywinski JB, et al. Health-related quality of life after coronary artery bypass grafting: a gender analysis using the Duke Activity Status Index. J Thorac Cardiovasc Surg 2004; 128: 284-95.

Rudski LG, Lai WW, Afilalo J, et al. Guidelines for the echocardiographic assessment of the right heart in adults: a report from the American Society of Echocardiography endorsed by the European Association of Echocardiography, a registered branch of the European Society of Cardiology, and the Canadian Society of Echocardiography. J Am Soc Echocardiogr 2010; 23: 685-713.

[15] Baig MK, Goldman JH, Caforio AL, et al. Familial dilated cardiomyopathy: cardiac abnormalities are common in asymptomatic relatives and may represent early disease. J Am Coll Cardiol 1998; 31: 195-201.

[16] Grunig E, Tasman JA, Kucherer H, et al. Frequency and phenotypes of familial dilated cardiomyopathy. J Am Coll Cardiol 1998; 31: 186-94.

[17] Michels VV, Moll PP, Miller FA, et al. The frequency of familial dilated cardiomyopathy in a series of patients with idiopathic dilated cardiomyopathy. N Engl J Med 1992; 326: 77-82.

[18] Bristow MR, Anderson FL, Port JD, et al. Differences in betaadrenergic neuroeffector mechanisms in ischemic versus idiopathic dilated cardiomyopathy. Circulation 1991; 84: 1024-39.

[19] White M, Roden R, Minobe W, et al. Age-related changes in betaadrenergic neuroeffector systems in the human heart. Circulation 1994; 90 : 1225-38.

[20] D'Andrea A, Salerno G, Scarafile R, et al. Right ventricular myocardial function in patients with either idiopathic or ischemic dilated cardiomyopathy without clinical sign of right heart failure: effects of cardiac resynchronization therapy. Pacing Clin Electrophysiol 2009; 32: 1017-29.

[21] Kashani A, Barold SS. Significance of QRS complex duration in patients with heart failure. J Am Coll Cardiol 2005; 46: 2183-92.

[22] Xiao HB, Roy C, Fujimoto S, Gibson DG. Natural history of abnormal conduction and its relation to prognosis in patients with dilated cardiomyopathy. Int J Cardiol 1996; 53: 163-70.

[23] Levy D, Bailey JJ, Garrison RJ, et al. Electrocardiographic changes with advancing age. A cross-sectional study of the association of age with QRS axis, duration and voltage. J Electrocardiol 1987; 20 (Suppl): 44-7.

[24] Brembilla-Perrot B, Beurrier D, Terrier de la Chaise A. Criteria of QRS duration in relationship to the age of myocardial infarction. Herz 1994; 19: 235-42.

[25] Bielecka-Dabrowa A, Mikhailidis DP, Hannam S, Aronow WS, Rysz J, Banach M. Statins and dilated cardiomyopathy: do we have enough data? Expert Opin Investig Drugs 2011; 20: 315-23.

[26] Bielecka-Dabrowa A, Goch JH, Mikhailidis DP, Rysz J, Maciejewski M, Banach M. The influence of atorvastatin on parameters of inflammation and function of the left ventricle in patients with dilated cardiomyopathy. Med Sci Monit 2009;15: MS12-23.

[27] Charlot M, Torp-Pedersen C, Valeur N, Seibæk M, Weeke P, Køber L. Anaemia and long term mortality in heart failure patients: a retrospective study. Open Cardiovasc Med J 2010; 4: 173-7.

[28] Struthers R, Erasmus P, Holmes K, et al. Assessing fitness for surgery: a comparison of questionnaire, incremental shuttle walk, and cardiopulmonary exercise testing in general surgical patients. Br J Anaesth 2008; 101: 774-80.

[29] Lindqvist P, Calcutteea A, Henein M. Echocardiography in the assessment of right heart function. Eur J Echocardiogr 2008; 9: 225-34.

This is an open access article licensed under the terms of the Creative Commons Attribution Non-Commercial License (http://creativecommons.org/licenses/ by-nc/3.0/) which permits unrestricted, non-commercial use, distribution and reproduction in any medium, provided the work is properly cited. 